\title{
Quantification of Thyroid Volume Using 3-D Ultrasound Imaging
}

\author{
Eva N. K. Kollorz, ${ }^{*}$ Dieter A. Hahn, Rainer Linke, Tamme W. Goecke, Joachim Hornegger, and Torsten Kuwert
}

\begin{abstract}
Ultrasound (US) is among the most popular diagnostic techniques today. It is non-invasive, fast, comparably cheap, and does not require ionizing radiation. US is commonly used to examine the size, and structure of the thyroid gland. In clinical routine, thyroid imaging is usually performed by means of 2-D US. Conventional approaches for measuring the volume of the thyroid gland or its nodules may therefore be inaccurate due to the lack of 3-D information. This work reports a semi-automatic segmentation approach for the classification, and analysis of the thyroid gland based on 3-D US data. The images are scanned in 3-D, pre-processed, and segmented. Several pre-processing methods, and an extension of a commonly used geodesic active contour level set formulation are discussed in detail. The results obtained by this approach are compared to manual interactive segmentations by a medical expert in five representative patients. Our work proposes a novel framework for the volumetric quantification of thyroid gland lobes, which may also be expanded to other parenchymatous organs.
\end{abstract}

Index Terms-3-D Ultrasound, Thyroid gland, Segmentation, Level set, Geodesic active contour.

\section{INTRODUCTION}

$\mathbf{T}$ HE thyroid gland belongs to the endocrine system and is localized in the neck just in front of the larynx. Diseases of the thyroid gland are among the most frequent endocrine disorders: In Germany, a large epidemiological study enrolling 96,278 subjects without prior thyroid disorder known yielded an incidence of $18.8 \%$ for goiter, i.e. pathological enlargement of the gland, and $23.4 \%$ for thyroid nodules [1]. This high incidence of thyroid abnormalities in Germany has been attributed to endemic iodine deficiency. In the USA, the population receives sufficient iodine supplementation via food. Surveys including such a large cohort of patients as in Germany are as yet missing in North America. However, the data available indicate a fairly high incidence of thyroid nodules also in the USA [2]. If untreated, goiter may lead to the formation of autonomous thyroid nodules, and possibly to hyperthyroidism. In the case of the detection of a thyroid nodule, thyroid cancer is one of the differential diagnoses and has to be ruled out with fine-needle biopsy, scintigraphy, and follow-up US [3]. US has

Manuscript received October 19, 2006; revised June 4, 2007. The Associate Editor responsible for coordinating the review of this paper and recommending its publication was M. Insana. Asterisk indicates corresponding author.

${ }^{*}$ E. N. K. Kollorz, D. A. Hahn, and J. Hornegger are at the Institute of Pattern Recognition (LME), Friedrich-Alexander-University ErlangenNuremberg, 91058 Erlangen, Germany (e-mail: Eva.Kollorz@informatik.unierlangen.de, phone: +49 (0) 9131/85-27894, fax: +49 (0) 9131/30 38 11).

R. Linke, and T. Kuwert are with the Department of Nuclear Medicine, University Hospital Erlangen, 91054 Erlangen, Germany.

T. W. Goecke is with the Department of Obstetrics and Gynaecology, University Hospital Erlangen, 91054 Erlangen, Germany.

Publisher Item Identifier become the most important acquisition technique for thyroid gland imaging [4], [5]. It is used quite frequently to screen for thyroid diseases since it allows volumetry of the whole gland, and its nodules and has a high sensitivity to detect thyroid tumors.

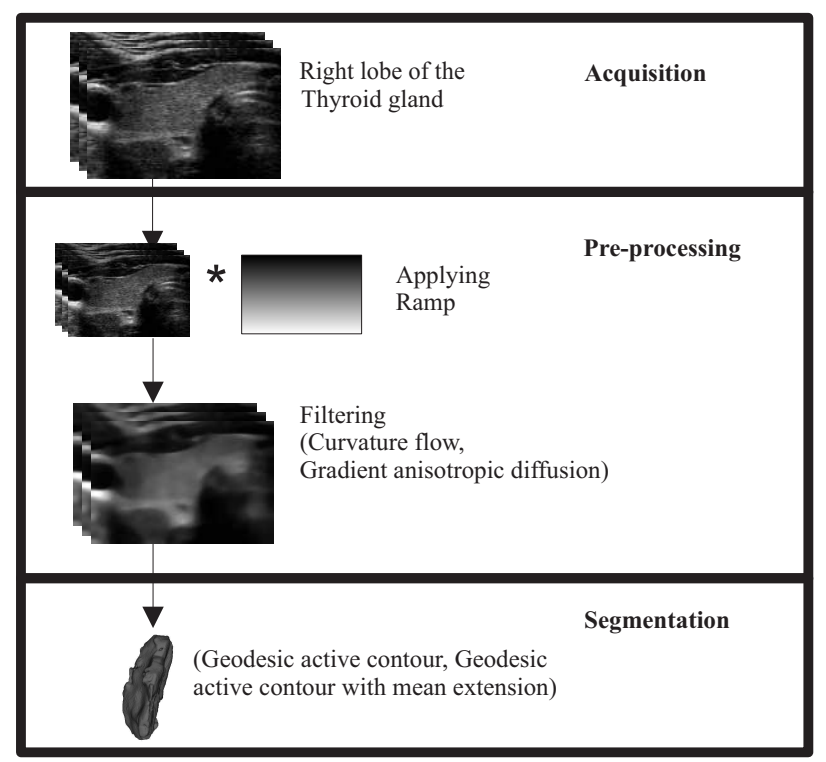

Fig. 1. Processing pipeline: The input for the pre-processing stage is the acquired 3-D US volume of one lobe of the thyroid gland. The volume is pre-processed by applying a linear ramp (to lift edges that are far away from the skin) and filtering. Subsequent segmentation is performed, which provides the 3-D mesh of the lobe of the thyroid.

Conventional sonography devices allow only for 2-D data acquisitions. For thyroid US, a probe is is placed onto the area of interest in the anterior neck. The physician then scans the thyroid gland by looking at a multitude of continuously varying 2-D images created by changing the position and orientation of the probe. This task involves the mental association of the 2-D images with the 3-D shape of the thyroid gland. At the end of the process, representative slices of the gland and/ or thyroid nodules are chosen for further measurements and diagnoses. The selection of these slices is rather subjective. Furthermore, in clinical routine, the volumetry of the thyroid gland or of its nodules is based on the measurement of crosssectional diameters on these slices, which are then entered into a formula assuming that the thyroid gland can be modeled by an ellipsoid. This assumption may in particular be violated in pathology. 2-D US has a low intra- as well as interobserver reproducibility [6], which may lead to problems in followup studies: It often cannot be reliably decided if a change 
TABLE I

IMAGING PROPERTIES OF THE USED VOLUMES: L STANDS FOR THE LEFT LOBE OF THE THYROID GLAND, R IS THE RIGHT ONE. THE SECOND COLUMN SHOWS THE VOLUME DIMENSIONS. THE SPACING OF A VOXEL IS MENTIONED IN THE THIRD COLUMN. GN STANDS FOR GAIN COMPENSATION, WHICH IS ADJUSTABLE AT THE US MACHINE AND WAS FIXED IN ALL EXPERIMENTS FOR SIMILAR IMAGE QUALITY AND GRAY VALUES (-8). THE SWEEP ANGLE OF THE DEDICATED 3-D PROBE WAS $29^{\circ}$ AND THE USED FREQUENCY CONSTITUTED $11.10 \mathrm{MHZ}$. ALL ACQUIRED VOLUMES HAVE 8 BIT DEPTH.

\begin{tabular}{|c|c|c|c|}
\hline $\begin{array}{c}\text { Data } \\
\text { set no. }\end{array}$ & Dimensions & $\begin{array}{c}\text { Voxel size } \\
{\left[\mathrm{mm}^{3}\right]}\end{array}$ & Parameter settings \\
\hline 1 & $149 \times 109 \times 199(\mathrm{~L})$ & 0.247389 & $11.10 \mathrm{MHz}, \mathrm{Gn}-8,29^{\circ}$ \\
2 & $149 \times 109 \times 199(\mathrm{R})$ & 0.247389 & \\
\hline 3 & $151 \times 101 \times 199(\mathrm{~L})$ & 0.245349 & $11.10 \mathrm{MHz}, \mathrm{Gn}-8,29^{\circ}$ \\
4 & $149 \times 109 \times 199(\mathrm{R})$ & 0.247389 & \\
\hline 5 & $145 \times 93 \times 199(\mathrm{~L})$ & 0.254016 & $11.10 \mathrm{MHz}, \mathrm{Gn}-8,29^{\circ}$ \\
6 & $149 \times 109 \times 199(\mathrm{R})$ & 0.247389 & \\
\hline 7 & $151 \times 101 \times 199(\mathrm{~L})$ & 0.245349 & $11.10 \mathrm{MHz}, \mathrm{Gn}-8,29^{\circ}$ \\
8 & $151 \times 101 \times 199(\mathrm{R})$ & 0.245349 & \\
\hline 9 & $149 \times 111 \times 199(\mathrm{~L})$ & 0.247134 & $11.10 \mathrm{MHz}, \mathrm{Gn}-8,29^{\circ}$ \\
10 & $151 \times 111 \times 199(\mathrm{R})$ & 0.246114 & \\
\hline
\end{tabular}

in the size of the examined structures is due to limitations in reproducibility of the employed method (due to a low reproducibility) or by pathology itself. It is well expected that the limitations of 2-D US imaging can be resolved through the use of 3-D US for thyroid gland analysis. Preliminary clinical data undermines this hypothesis [7]. Nowadays, 3-D US scanning devices are increasingly used within the clinical routine. The data acquired can be voxelized, which provides the basis for a more detailed study of the thyroid gland based on both state-of-the-art volume rendering and medical image processing techniques. For the physician, it is now possible to replicate the diagnosis without re-scanning the patient. By incorporating additional 3-D information, the diagnosis may become largely observer-independent and accurate volumetric measurements become possible. This work proposes a semiautomatic segmentation approach for the classification and analysis of thyroid glands based on 3-D US data. Fig. 1 illustrates the image processing pipeline used in our framework. First, a 3-D US scan of a thyroid lobe is acquired with a dedicated 3-D probe and without any additional tracking device. Usually one scan is sufficient to acquire an entire lobe as we set the field of view (FOV) large enough to depict each entire lobe. This volume is the input for the pre-processing stage. The pre-processing module consists of an intensity correction step and additional filtering for noise reduction. Our main focus lies on the complete acquisition of each lobe of the thyroid glands as an entire US volume. We concentrate on the accuracy of the thyroid gland volume estimation. Finally, the volume becomes the input for the segmentation step which is based on level set methods. The result of the processing pipeline is a 3-D mesh of the according thyroid lobe.

\section{STATE-OF-THE-ART}

\section{A. Pre-processing}

Our pre-processing involves intensity correction and filtering. For the processed types of images, intensity correction is necessary because the energy of the sound waves degrades with increased distance from the probe. This results in similar amplitudes at the lower border of the lobe of the thyroid gland. The input for the segmentation is a speed image, which is based on the gradient magnitude strength and a sigmoid mapping. Therefore, the amplitudes are lifted at positions farther away from the probe to boost the gradient magnitude strength. Though a research in this field is not extensively published, intensity correction is an active topic of research. For example, Xiao et al. perform intensity inhomogeneity correction, including segmentation in one step (breast, cardiac images) [8]. They use a combination of the maximum a posteriori (MAP) and Markov random field (MRF) methods.

In comparison, filtering operations for noise reduction are much more widely used in US images. Mean and median filtering are commonly employed. More complex operations include the "sticks" filter (short line segments) [9], [10] or various types of diffusion filters [11], [12], [13], [14]. Calóope et al. [15] provide a comparison of filtering operations for US images. They present and evaluate the mean, median, Frost and MAP filter and also the applicability of the wavelet transformation for this task. They conclude that the modified MAP filter performed best for their purpose. Fu et al. [12] suggest an adaptive anisotropic diffusion approach for US image denoising and edge enhancement, which is coherent with the results of Krissian et al. [13]. Yu and Acton [14] compare non-linear anisotropic diffusion with adaptive speckle filters (Lee and Frost filter). According to Tauber et al. [16] widely used speckle reduction filters are the Lee, Frost, Kuan and Gamma Maximum a Posteriori (GMAP) filter. However, they applied the anisotropic diffusion filter for the results in their publication. Betrouni et al. [17] use an adapted noise filter. Fieberg [18] mentions step mean, Gaussian, median, adaptive and morphological filtering operations. Though some US machines, (e.g. the used General Electric Voluson 730 Expert) provide Speckle Reduction Imaging (SRI), which is only displayed and not accessible in the stored volumes. To our knowledge, there exist no publications about the details of the implemented methods within the commercially available US machines.

\section{B. Segmentation}

The segmentation of the thyroid US images falls into the category of small anatomical object segmentation from US images like the prostate (using Transrectal US (TRUS) [19], [20]), tumors in the breast [21], the carotid artery [22] or the ischemic myocardium. Many popular methods for segmenting such anatomical structures are based on level sets [17], [21], [23], [24]. For example, Betrouni et al. [17] use elastically deformable models for the segmentation of the prostate. Overhoff et al. [25] make use of a region-based approach for visualization of anatomical structures of the liver. Region growing with thresholding and live-wire/live-lane methods are used by Fieberg [18] in addition to morphological segmentation methods. Sarti et al. [24] introduce a maximum 
likelihood segmentation of US images with an underlying Rayleigh distribution model. The active contour model is also applied in Chen et al. [21] to segment tumors within the breast. Several segmentation methods for US images are mentioned in Abolmaesumi and Sirouspour [23], e.g. texture operators and mathematical morphology.

Thyroid volumetry in 3-D US is extensively addressed in Schlögl et al. [7]. They applied an ellipsoid model for volume measurements, manual segmentation and multiplanar volume approximation (MVA) on slices extracted by a 2-D US device. A manual volume measurement of the thyroid with 3-D US with MVA is, for instance, discussed in [26], [27]. Thyroid volume measurement with conventional 2-D US is also addressed in [28], [29]. Compared to 2-D US, the segmentation of 3-D US volumes is still rarely used for clinical purposes. Overhoff et al. [25] apply 3-D US for the visualization of structures in the liver (region-based approach). Baillard et al. [22] use 3-D US for the segmentation of the carotid artery (level sets). The thyroid gland segmentation has been addressed in [7] by a manual segmentation approach.

\section{Material AND Methods}

\section{A. Image Acquisition Protocol}

The scanning of the thyroid glands for this work has been performed with a 3-D General Electric (GE) Voluson 730 Expert US system. A 5 to $12 \mathrm{MHz}$ probe (RSP5-12 wide band linear volume probe) was used for the acquisition, which resulted in a penetration depth range of approximately 5 to 10 $\mathrm{cm}$. The probe should be held steady during the 3-D scan process, because during the scanning a fan of images is acquired by an automatically rotating transmitter. The scanning process for one lobe of the thyroid gland takes about five seconds. The volumes are transferred into the cartesian coordinate system and saved in DICOM format. In general, one 3-D volume was acquired for each lobe. Details on the image acquisition protocol for the individual volumes can be found in Table I. The time-gain compensation of the US machine was adjusted by a medical expert before we acquired the thyroid images.

In literature, US image artifacts are mainly characterized in terms of speckle and noise. The noise contribution is vastly described by additive models, whereas for speckle, the models can be multiplicative [11], [12], [14] or signal-dependent [13], [16], [30]. The latter types even include noise aspects as well, which is the reason why they are also mentioned as speckle noise models. Therefore, we evaluated the pre-processing filters with both: A Rayleigh distributed signal-dependent speckle noise model $x=s+n \sqrt{s}$ ( $s$ : Original signal, $n$ : Noise, $x$ : Noisy signal) and a Rayleigh distributed multiplicative speckle model $x=s * n$ ( $n$ : Multiplicative speckle noise).

\section{B. Pre-processing of US volumes}

This subsection covers the application of a linear ramp as intensity correction and different edge-enhancing diffusion filters to reduce the noise in the acquired US volumes.
1) Applying a linear ramp: Inherent to US imaging is the existence of a "bias field" that affects the image amplitudes. This field appears as gradually decreasing log amplitudes with increasing distance from the probe. This may affect segmentation approaches that use amplitude information. However, defining a statistical description for the tissue in the area of interest is not a trivial task. The problem is that the number of tissue classes, mean and standard deviation of their amplitude values may vary in great extent. Histogram equalization did not provide any promising results, either. Hence, a simple method was devised: The multiplication of a linear intensity ramp in order to approximately compensate for the log amplitude decrease. This ramp function is used to lift the amplitudes further away from the skin tissue where the probe is located and cannot be handled by the time-gain compensation. The ramp intensities are in the range of $[1,2]$ and multiplied with the image amplitudes. This is done slice by slice. The range of the ramp was determined heuristically to improve edges at inner structures, e.g. the lower border of the thyroid lobe.

2) Edge-enhancing diffusion: Due to the low image quality of US data in general, it is important to reduce noise and speckle in nearly homogeneous regions, while preserving edges. We implemented and evaluated several filtering approaches that have been proposed in the literature. In the following section, we describe two representative edgeenhancing diffusion filters which were developed specifically for combining noise reduction with edge preservation.

\section{Curvature flow filter (CF)}

The CF filter performs edge-preserving smoothing similar to the classical anisotropic diffusion. The filter uses a level set formulation, where the isointensity contours in an image are treated as level sets [31]. The functional is then evolved under the control of a diffusion equation with a speed that is proportional to the curvature of the contour:

$$
\frac{\partial I}{\partial t}=\kappa\|\nabla I\|
$$

with $\kappa$ denoting the curvature and $\nabla$ the gradient operator. To derive a numerical algorithm, one has to consider the discretization of time $t=n \tau$, where $n \in \mathbf{N}$ is the number of iterations and $\tau$ the time step. This leads to

$$
\frac{I^{n+1}-I^{n}}{\tau}-\kappa\left\|\nabla I^{n}\right\|=0 .
$$

Large time steps result in a greater and faster smoothing. However, the time step has to be chosen carefully for numerical stability reasons. Regions with high curvature values will diffuse faster compared to regions with low curvature. Small jagged noise artifacts will therefore disappear more quickly, whereas large scale interfaces will slowly evolve. Sharp boundaries between objects are preserved.

Gradient anisotropic diffusion filter (GAD) 
In GAD the diffusion scheme is given by:

$$
\frac{\partial I}{\partial t}=\operatorname{div}(c \nabla I)
$$

where $c$ is the diffusivity of the equation and also called diffusion coefficient [32], [33]. $I$ is treated as $I(\boldsymbol{x}, t), \boldsymbol{x} \in \mathbf{R}^{2}$, i.e. the two-dimensional image $I(\boldsymbol{x})$ at a certain time $t>0$. The initial condition is:

$$
I(\boldsymbol{x}, 0)=I_{0}(\boldsymbol{x}),
$$

where $I_{0}$ is the original image. The diffusion equation becomes non-linear, e.g. with the following diffusivity:

$$
c=e^{-\frac{\|\nabla I\|^{2}}{2 k^{2}}},
$$

where $k$ is the conductance parameter that allows for controlling the amount of diffusion near edges in the image. The larger the values for $k$, the less weight is put on the gradient magnitude.

\section{Segmentation methods}

Our ultimate goal is to automatically segment the entire 3-D volume of the thyroid lobe. In an early stage of our research, we applied region growing and watershed based segmentation with little success. Thresholding techniques can also not deal with the poor image quality and the bias field. Level set methods on the contrary, allow for more flexibility and yield promising results. In this section we will briefly introduce the geodesic active contour and the proposed extension for the US volumes we are dealing with.

1) Geodesic active contour (GAC): GAC methods [34] are based on level sets that are briefly illustrated in the following. In the general $N$-dimensional case, the interface $\Gamma$ is represented by the zero level set of the function $\Phi(\boldsymbol{x}, t)$ : $\mathbf{R}^{N} \times[0, \infty) \rightarrow \mathbf{R}$ such that

$$
\begin{aligned}
\Gamma & =\left\{\boldsymbol{x} \in \mathbf{R}^{N}, t \in[0, \infty): \Phi(\boldsymbol{x}, t)=0\right\}, \\
\Gamma_{i} & =\left\{\boldsymbol{x} \in \mathbf{R}^{N}, t \in[0, \infty): \Phi(\boldsymbol{x}, t)<0\right\}, \\
\Gamma_{o} & =\left\{\boldsymbol{x} \in \mathbf{R}^{N}, t \in[0, \infty): \Phi(\boldsymbol{x}, t)>0\right\},
\end{aligned}
$$

where $\Gamma_{i}$ describes the region inside and $\Gamma_{o}$ the region outside of the interface $\Gamma$ (for 2-D see Fig. 2). The level set function evolves over time and leads to the partial differential equation (PDE):

$$
\frac{\partial \Phi}{\partial t}=F\|\nabla \Phi\|,
$$

where $F$ is a force term responsible for growing or shrinking and $\nabla \Phi$ the gradient of the level set function.

The equation for geodesic active contours is given by:

$$
\frac{\partial \Phi}{\partial t}=(\gamma Z(\boldsymbol{x}) \kappa-\lambda P(\boldsymbol{x}))\|\nabla \Phi\|-\alpha(-\nabla P(\boldsymbol{x}))^{T} \nabla \Phi
$$

where $(-\nabla P(\boldsymbol{x}))$ is the advection vector field, $P(\boldsymbol{x})$ the propagation speed and $Z(\boldsymbol{x})$ (curvature speed) a spatial modifier term for the mean curvature $\kappa . \alpha, \lambda$ and $\gamma$ are scalar weighting factors. The mean curvature, $\kappa$, in 3-D is given by:

$$
\kappa=-\nabla^{T} \cdot \frac{\nabla \Phi}{\|\nabla \Phi\|}
$$

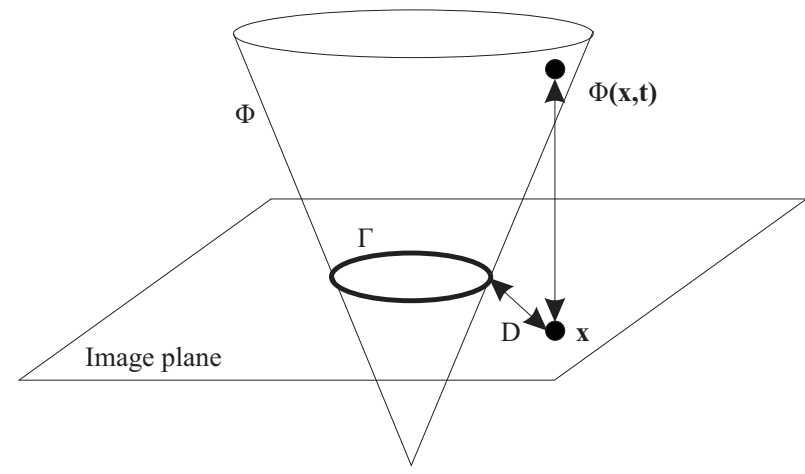

Fig. 2. Level set algorithm illustration (2-D). The 2-D curve $\Gamma$ is represented by a 3-D level set function $\Phi(\boldsymbol{x}, t)$ which intersects the image plane. The value of $\Phi$ at some point $\boldsymbol{x}$ is defined as its distance D to the curve $\Gamma$. Positive distance values are inside the curve, negative ones outside the curve. The curve evolves over time $t, t=0$ is the initialization.

In our experiments, we set the curvature speed equal to the propagation speed. The term contained within the first brackets of Eq. 8 corresponds to the force $F$. The interface grows if the force $F$ is negative and vice versa.

The propagation of the curve is driven by a speed image that is based on the gradient magnitude image and obtained via the sigmoid function:

$$
\hat{I}(\boldsymbol{x})=\left(\hat{I}_{\text {max }}-\hat{I}_{\text {min }}\right) \cdot \frac{1}{1+e^{\left(\frac{I(\boldsymbol{x})-\zeta}{\rho}\right)}}+\hat{I}_{\text {min }},
$$

which results in a non-linear mapping of the image amplitudes. $I(\boldsymbol{x})$ denotes the image amplitude at position $\boldsymbol{x}, \hat{I}(\boldsymbol{x})$ the resulting intensity and $\hat{I}_{\max }\left(\hat{I}_{\min }\right)$ the maximum (respectively minimum) of the desired output intensity. Parameters $\rho$ and $\zeta$ $\in \mathbf{R}$ are used to intensify the differences between regions of low and high values in the speed image. In the ideal case, the speed should be maximal in homogeneous areas and minimal near edges. To determine values for $\rho$ and $\zeta$, the gradient magnitude should be considered. $\zeta$ defines the intensity center for the sigmoid function and $\rho$ its width. Let $c_{\min }$ be the minimum intensity value along the boundary that surrounds the structure and $\bar{c}$ the mean value within the structure. These two values indicate the dynamic range that has to be mapped into the intensity interval of the speed image (e.g. $[0,1])$. The suggested values for $\zeta$ and $\rho$ may then be:

$$
\begin{aligned}
\zeta & =\frac{c_{\text {min }}+\bar{c}}{2} \\
\rho & =\frac{\bar{c}-c_{\text {min }}}{6} .
\end{aligned}
$$

This yields a speed image with minimal values in regions with a large gradient magnitude and maximal values in areas with vanishing gradients. $P(\boldsymbol{x})$ therefore acts as a speed parameter that lets the interface evolve more quickly in homogeneous regions and slows down at edges.

$\alpha, \lambda$ and $\gamma$ are scalar weighting factors that influence the evolution of the zero level set. The parameter $\alpha$ scales the advection vector field that influences the interface near edges in the image. The advection vector field ideally prevents the level set from leaking into adjacent structures by providing an inward pointing force. The propagation scaling $\lambda$ controls the 
influence of the propagation speed. The contour or surface will grow if the value is positive, otherwise the interface will shrink. $\gamma$ weights the magnitude of the curvature values that are calculated on the evolving interface. Large values relative to the other parameters will lead to a smooth interface. The interface slows down at areas with high curvature (sharp edges) and may end up smooth at sharp corners. Due to the computation time, a sparse field approach is used to update the level set equation.

The root mean squared change (RMSC) of the curve is used as a convergence criterion. The evolution of the interface converges if the RMSC is below a specified threshold. A small threshold value for the RMSC will result in a tight fit of the curve, but will take more computations. In our experiments, we used a RMSC of 0.05 as stopping criterion. The initial contour is propagated outwards until it sticks to the shape boundaries. This process is guided by an edge potential map following the approach by Malladi et al. [35]. Finally, an input image defines the initialization of the level set, which may consist of seed points or an initial curve.

2) Geodesic active contour with mean extension (GACM): For the specific case of the thyroid 3-D US images, we suggest the following intensity based extension of the level set equation:

$$
\begin{aligned}
\frac{\partial \Phi}{\partial t} & =(\gamma Z(\boldsymbol{x}) \kappa-\lambda P(\boldsymbol{x}))\|\nabla \Phi\|-\alpha(-\nabla P(\boldsymbol{x}))^{T} \nabla \Phi \\
& +\eta\left(I-\mu_{0}\right)^{2},
\end{aligned}
$$

where $I(\boldsymbol{x})$ denotes the intensity of the pre-processed volume, $\mu_{0}$ the mean intensity calculated in the initialization step and $\eta$ a weighting factor for the intensity regularization. This additional term penalizes evolutions of the curve towards image regions that deviate from the initial intensity mean value of the anatomical structure of interest. The motivation behind the introduction of this extension term is based on the typical intensity appearance of the thyroid images: Usually the intensity gradient between the thyroid and trachea regions decreases with increasing distance from the probe. The edge potential in critical areas may therefore be insufficient to keep the interface within the thyroid region. This may result for example in a curve which leaks into the trachea. The proposed formulation ideally suppresses any leakage at low-contrast edges and will be referred to as GACM for the remainder of this article.

\section{EXPERIMENTAL RESULTS}

Since the accuracy of our segmentation depends on the effectiveness of our pre-processing steps, we first separately evaluated the ability of our proposed pre-processing filters in reducing noise and speckle while preserving edge information. In the following the term noise is used to denote speckle and noise. The evaluation for the segmentation itself is presented in the next subsection.

Table II shows the required runtimes for the different steps in the image processing chain on an AMD Athlon XP $3200+, 2.20 \mathrm{GHz}$ with $1 \mathrm{~GB}$ RAM.

TABLE II

RUNTIMES FOR THE OPERATIONS USED IN THE PRESENTED IMAGE PROCESSING CHAIN. VALUES STEM FROM A VOLUME OF DIMENSIONS $(149 \times 109 \times 199)$.

\begin{tabular}{|l|r|}
\hline Operation & Runtime [s] \\
\hline Reading DICOM series & 1.625 \\
Applying intensity ramp & 0.204 \\
CF filtering (iterations $=20, \tau=0.2$ ) & 28.000 \\
GAD filtering (iterations $=20, \tau=0.0625, k=3$ ) & 66.391 \\
GAC Segmentation (iterations $=395$ ) & 137.828 \\
Writing volume & 0.281 \\
\hline
\end{tabular}

\section{A. Pre-processing}

1) Evaluation criteria: The criteria for the evaluation of the pre-processing are: The mean squared error (MSE), the signal-to-MSE ratio (S/MSE) and the edge preservation $\beta$ [15]. The reduction of noise is analyzed with MSE and S/MSE. For specifying the reduction assuming a multiplicative speckle model, the input for S/MSE are the log transformed images. The 2-D phantom image $G$ (size: $256 \times 256$ ) was corrupted with noise to create artificial images $\hat{G}$ for the evaluation of the different filtering methods. This evaluation is performed for simplicity on 2-D images, but the results generalize on higher dimensions.

The MSE is defined by:

$$
\mathrm{MSE}=\frac{1}{N} \sum_{i=1}^{N}\left(\hat{G}_{i}-G_{i}\right)^{2},
$$

where $N$ is the number of elements in the image, $i$ the $\mathrm{i}$-th image point. If all noise is reduced (ideal case), MSE would be zero.

The S/MSE is defined by:

$$
\mathrm{S} / \mathrm{MSE}=10 \cdot \log _{10}\left(\sum_{i=1}^{N} G_{i}^{2} / \sum_{i=1}^{N}\left(\hat{G}_{i}-G_{i}\right)^{2}\right)
$$

and the edge preservation $\beta$ :

$$
\beta=\frac{\Gamma\left(G_{\Delta}-\bar{G}_{\Delta}, \hat{G}_{\Delta}-\overline{\hat{G}}_{\Delta}\right)}{\sqrt{\Gamma\left(G_{\Delta}-\bar{G}_{\Delta}, G_{\Delta}-\bar{G}_{\Delta}\right) \cdot \Gamma\left(\hat{G}_{\Delta}-\overline{\hat{G}}_{\Delta}, \hat{G}_{\Delta}-\overline{\hat{G}}_{\Delta}\right)}}
$$

where $G_{\Delta}$ expresses the high-pass filtered version of $G$ with $3 \times 3$ Laplacian filter and $\bar{G}$ is the mean of the image, respectively. This filtering detects the edges in the two images $G, \hat{G}$, which is an important factor for the performance of the segmentation. Equation (14) is closely related to the correlation coefficient. The correlation coefficient is 1 for an increasing linear relationship, 0 for no linear relationship and values in between indicate the degree of linear dependence between the two variables (here: $G, \hat{G}$ ). If all edges in the pre-processed, artificially generated and noisy phantom image 
are preserved, $\beta$ converges to one. Given two images $G$ and $J$, the function $\Gamma$ is defined as:

$$
\Gamma(G, J)=\sum_{i=1}^{N} G_{i} \cdot J_{i} .
$$

Equations (13) and (14) are crucial for the evaluation of filtering operations on US data, as noise has to be reduced in homogeneous areas, whereas edges are to be preserved. These criteria are particularly important for the processing of the images with gradient-based segmentation approaches.

TABLE III

EVALUATION OF TWO DIFFERENT EDGE-ENHANCING DIFFUSION FILTERS: CURVATURE FLOW (CF) AND GRADIENT ANISOTROPIC DIFFUSION (GAD).

THE FIRST SECTION SHOWS THE RESULTS FOR A SIGNAL-DEPENDENT SPECKLE NOISE MODEL. THE SECOND SECTION EVALUATES A MULTIPLICATIVE SPECKLE MODEL. EVALUATION CRITERIA ARE: MEAN SQUARED ERROR (MSE), SIGNAL-TO-MSE RATIO (S/MSE) AND EDGE PRESERVATION $\beta$. CF SHOWS THE BEST RESULTS FOR ALL THREE EVALUATION CRITERIA. GAD PRESENTS AVERAGELY LESS EDGE PRESERVATION THAN CF. $\mu$ DENOTES THE MEAN OF THE NOISE AND $\sigma^{2}$ THE VARIANCE.

\begin{tabular}{|c|c|c|c|}
\hline $\begin{array}{l}\text { Filter method and } \\
\text { parameter settings }\end{array}$ & MSE & S/MSE [db] & $\beta$ \\
\hline Best case: & Min (0) & Max & Max (1) \\
\hline \multicolumn{4}{|c|}{ Signal-dependent speckle noise model $\left(\mu=0.2100, \sigma^{2}=0.0291\right)$} \\
\hline \multicolumn{4}{|c|}{\begin{tabular}{|l|l|l|l} 
Curvature flow & & &
\end{tabular}} \\
\hline iterations $=10, \tau=0.2$ & 0.0021 & 18.6594 & 0.6547 \\
\hline iterations $=20, \tau=0.2$ & 0.0016 & 19.9542 & 0.7105 \\
\hline iterations $=30, \tau=0.2$ & 0.0014 & 20.5563 & 0.7117 \\
\hline \multicolumn{4}{|l|}{ Gradient anisotropic diffusion } \\
\hline iterations $=10, \tau=0.125, k=4$ & 0.0016 & 19.8725 & 0.5516 \\
\hline iterations $=20, \tau=0.125, k=4$ & 0.0016 & 19.7088 & 0.4659 \\
\hline iterations $=20, \tau=0.125, k=1$ & 0.0067 & 13.6172 & 0.2131 \\
\hline \multicolumn{4}{|c|}{ Multiplicative speckle model $\left(\mu=0.7496, \sigma^{2}=0.1145\right)$} \\
\hline \multicolumn{4}{|c|}{\begin{tabular}{l|l|l|l} 
Curvature flow & & &
\end{tabular}} \\
\hline iterations $=10, \tau=0.2$ & 0.0064 & 17.4878 & 0.4042 \\
\hline iterations $=20, \tau=0.2$ & 0.0051 & 18.5638 & 0.5380 \\
\hline iterations $=30, \tau=0.2$ & 0.0044 & 18.9140 & 0.5773 \\
\hline \multicolumn{4}{|l|}{ Gradient anisotropic diffusion } \\
\hline iterations $=10, \tau=0.125, k=4$ & 0.0088 & $\begin{array}{l}15.6476 \\
16.4090\end{array}$ & $\begin{array}{l}0.0866 \\
0.0857\end{array}$ \\
\hline $\begin{array}{l}\text { iterations }=20, \tau=0.125, k=4 \\
\text { iterations }=20, \tau=0.125, k=1\end{array}$ & 0.0227 & 9.3240 & 0.0599 \\
\hline \multicolumn{4}{|l|}{$\bar{\tau}$ : Time step, $k$ : Diffusivity } \\
\hline
\end{tabular}

2) Results: The noise reduction as well as the edge preservation properties of different filters for US imaging are evaluated with three criteria: Mean squared error (MSE), signal-to-MSE ratio (S/MSE) and the edge preservation $\beta$. Several techniques were implemented and evaluated. Representatively, the two filters with the best results (curvature flow, gradient anisotropic diffusion) are itemized in Table III.

GAD shows the same tendency for the signal-dependent speckle noise model and the multiplicative speckle model, only with different range. $\beta$ has considerable lower, MSE higher values for the speckle model than for the noise model. It can be seen that $\mathrm{CF}$ filtering with 20 iterations is sufficient for high edge preservation and high S/MSE values. 30 or more iterations only result in higher computational times but

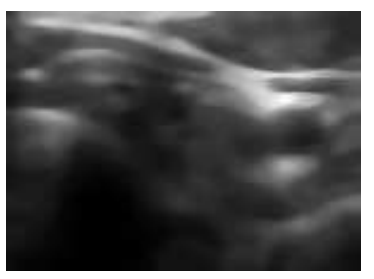

(a)

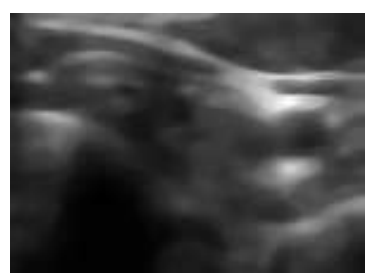

(c)

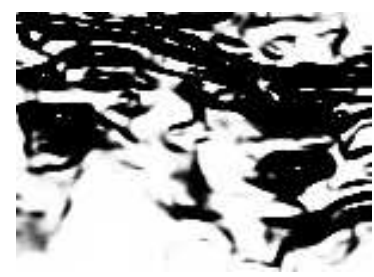

(b)

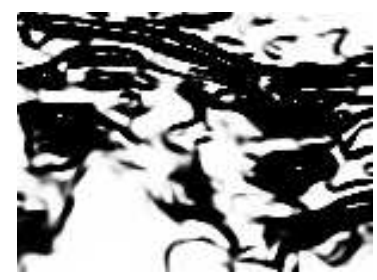

(d)
Fig. 3. Subfigure 3(a) and 3(c) are pre-processed images. The right column shows the speed images which are important for the segmentation. The first row shows results without applying the ramp, the second row with. The edge at the lower right boundary of the lobe was increased. The intensity increase can be seen in subfigure 3(c).

nearly identical values for S/MSE and $\beta$.

To acquire the presented results, the settings for filtering the real US data were chosen as follows: For GAD 20 iterations, time step $\tau=0.0625$ and $k=3$; for CF 20 iterations and a time step of $\tau=0.2$. The lower time step for GAD depends on the expansion from 2-D (phantom image) to 3-D (US volumes). Otherwise the problem would no longer be numerically stable or a more advanced numerical solver is needed.

Fig. 3 motivates the application of the ramp. It can be seen that far away lying edges to the skin are increased by multiplying a linear ramp. Some former experiments showed that this procedure prevents from leaking. The applied pre-processing filter for both cases in Fig. 3 is the CF filter.

\section{B. Segmentation}

1) Evaluation criteria: For evaluating the segmentation, the standardized measurements of sensitivity and specificity were applied. The sensitivity (SE, true positive rate) reflects the probability of classifying voxels as thyroid gland tissue (C) given that the tissue actually belongs to the thyroid gland (T), whereas the specificity is the true negative rate:

$$
\begin{aligned}
& \mathrm{SE}=P(C \mid T) \\
& \mathrm{SP}=P(\bar{C} \mid \bar{T}) .
\end{aligned}
$$

We compared the manually segmented 3-D data sets to the results of the proposed semi-automatic segmentations. Each manual segmentation was realized with the Random Walker approach by Grady [36]. One segmentation for one thyroid lobe takes approximately 5 minutes. For a direct comparison of the results, the surfaces of the segmented structures are extracted and represented as triangular meshes. To apply the commonly used Hausdorff distance (HD) measures, the meshes are regarded as point clouds with points located on the 
vertices. Given two point sets $A$ and $B$, the directed Hausdorff distance is given by:

$$
h(A, B)=\sup _{\boldsymbol{a} \in A}\left\{\inf _{\boldsymbol{b} \in B}\{d(\boldsymbol{a}, \boldsymbol{b})\}\right\},
$$

where $\boldsymbol{a}$ and $\boldsymbol{b}$ are the points of the meshes and $d$ is the Euclidean distance. Eq. 16 describes the maximal distance of a point set $A$ to the nearest point within the set $B$. As $h$ is not symmetric (i.e. $h(A, B) \neq h(B, A)$ ), the general Hausdorff distance may be used as its symmetric equivalent:

$$
H(A, B)=\sup \{h(A, B), h(B, A)\} .
$$

The distances are calculated in physical coordinates (mm). Additionally, the mean distance errors $\overline{h(A, B)}, \overline{h(B, A)}$ are calculated.

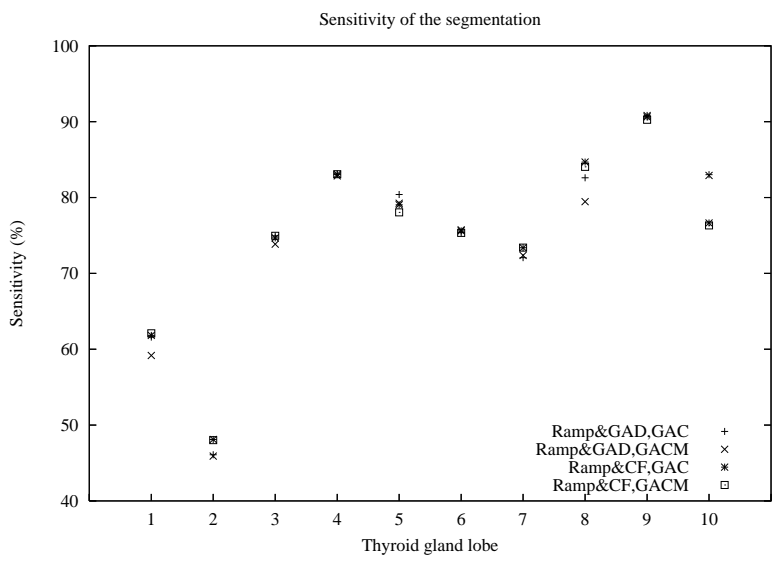

Fig. 4. Sensitivity (SE) of the 10 segmented thyroid lobes. Different point types show the combination of the presented pre-processing and segmentation methods.

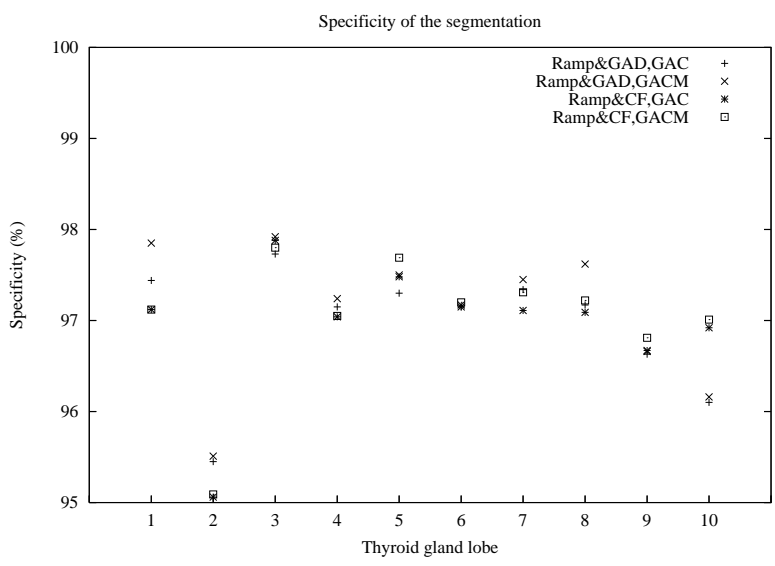

Fig. 5. Specificity (SP) of the 10 segmented thyroid lobes. Different point types show the combination of the presented pre-processing and segmentation methods.

2) Results: To initialize the expanding level set formulations, five spherical volume elements with a specific radius were chosen as seed for each thyroid lobe. In the following we compare the results obtained from different combinations between the pre-processing and the

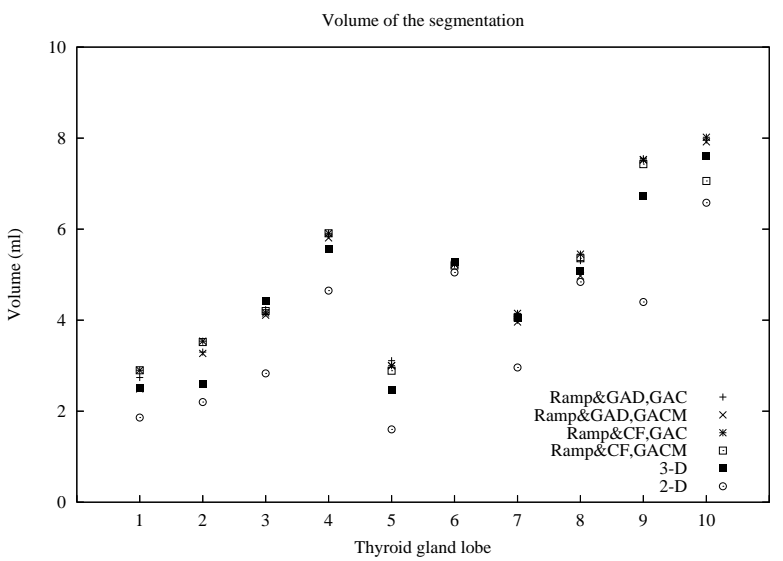

Fig. 6. Volume in milliliters of the 10 segmented thyroid lobes. 2-D describes the volume measurement based on the standard ellipsoid formula $0.52 \times$ length $\times$ width $\times$ depth as performed by the medical expert. 3-D means the ground truth segmentation of the nuclear medicine expert in 3-D.

segmentation approaches. In summary, we compare four combinations of $\mathrm{GAD}, \mathrm{CF}$ as pre-processing algorithms with GAC and GACM as segmentations. As described earlier, an additional contrast-enhancement by the application of a linear ramp has always led to better segmentation results. Therefore, the ramp filtering was always applied. Fig. 4 and 5 show the sensitivity and specificity of the segmentation results for each combination. For the sensitivity there is a trend that all combinations perform similar. The segmentation with the mean extension provide advantages in case of specificity. Although the sensitivity is above $75 \%$ for most of the lobes, the first two segmentation results (especially for lobe number two) exhibit less quality. One slice of the lobe of thyroid gland number two can be seen in Fig. 8 that shows the difficulty in finding appropriate edges of the thyroid. A strong anisotropic smoothing is necessary in this case to eliminate noisy heterogeneous structures inside the thyroid region. This thyroid gland image features very low edge contrast in general, which results in a low edge strength. In order to circumvent a leakage of the segmentation at the thyroid boundaries, the edge strength parameters of the level set formulation have to be adapted. This restriction in general leads to a slowly evolving level set interface and in our case to less segmented thyroid tissue. The concurrence of these factors ends in a low sensitivity percentage similar applies for the thyroid lobe number one. The specificity is around $97 \%$ for nearly all data sets. In our experiments, a combination of the initial ramp together with a curvature flow pre-processing and the proposed GACM segmentation yield satisfactory results. Fig. 7 presents the 3-D manual hand segmentation compared to the different results of the various pre-processing and segmentation combinations applied to the data set number five. The sensitivity percentage for this data set is between $78 \%$ and $80 \%$. A comparison between the manual and semi-automatic segmentation shows that the isthmus is usually not segmented in the semi-automatic approach due to its small and narrow shape. For the proposed segmentation algorithms, this challenging problem generally leads to less 


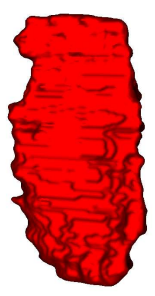

(a)

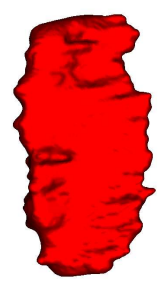

(f)

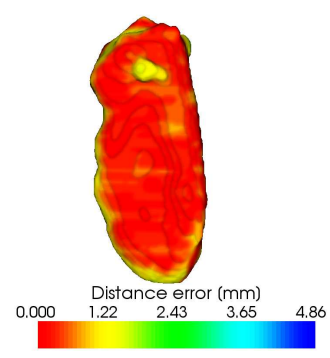

(b)

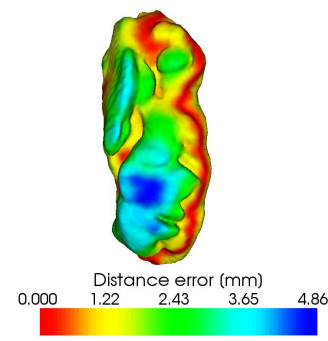

(g)

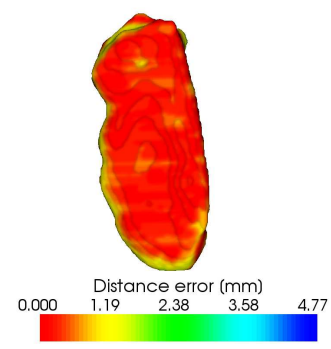

(c)

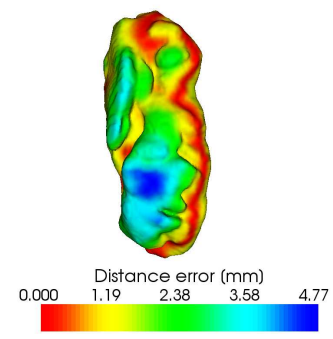

(h)

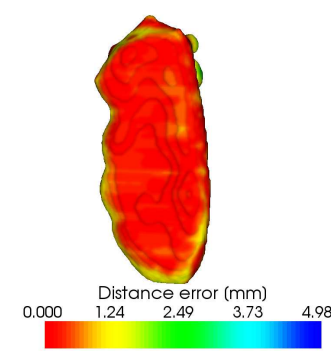

(d)

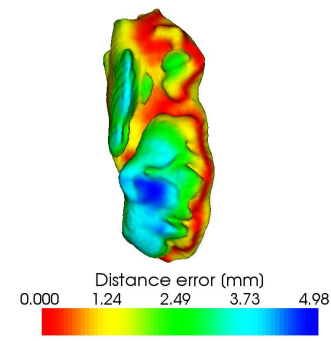

(i)

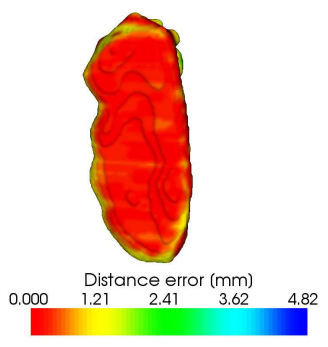

(e)

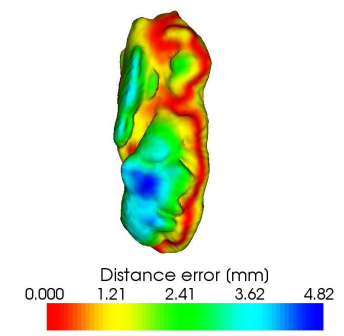

(j)

Fig. 7. Subfigure 7(a) and 7(f) show the manual ground truth segmentation of the nuclear medicine physician of thyroid gland lobe number five. Different segmentation results are visualized via Hausdorff distance: 7(b) and 7(g) are results of the ramp, GAD and GAC combination. Subfigure 7(c) and 7(h) visualize the combination of ramp, GAD and GACM. Ramp, CF and GAC is shown in 7(d) and 7(i), and 7(e) and 7(j) with GACM. The upper row shows the front view, the lower the back.

sensitivity values compared to the manual reference that often includes parts of the isthmus. This fact has to be considered while analyzing the sensitivity percentages given in Fig. 4. Subfigure 7(b) depicts already leakage at the anterior surface of the thyroid lobe. This is prevented by the GACM approach (see Fig. 7(c)). Fig. 7(g) and 7(h) show that the segmentation leaks for GAD pre-processing in the middle of the posterior surface. It can also be seen that $\mathrm{CF}$ provides better segmentation results with respect to posterior side of the thyroid.

Fig. 6 illustrates the different volumes in milliliters, where the 2 -D ellipsoid formula $(0.52 \times$ length $\times$ width $\times$ depth [28]), the 3-D ground truth segmentation and the semi-automatic segmentations of the different combinations are compared to each other. It is obvious that the 2-D method systematically underestimates the actual thyroid volume. We also suggest in concurrence with Reinartz et al. [28] that the correction factor of 0.52 for the 2-D based volume measurements has to be revised.

Table IV shows the Hausdorff distances of the semiautomatic segmented volumes $(\mathrm{S})$ to the manual ground truth segmentations $(\mathrm{M})$ in both directions.

\section{Discussion}

In general, the image quality of 3-D US is quite poor and it is hard to provide desirable image enhancements by traditional pre-processing techniques. However, the experiments presented in this article show promising results. They demonstrate that the current clinical approach towards 3-D volume measurements of the thyroid volume using 2-D US and Brunn's formula is clearly outperformed by the presented semi-automatic segmentation algorithms. This is proven by a detailed comparison with 3-D manually segmented volumes used as gold standard references. The evaluation of the sensitivity and specificity for the presented algorithms shows advantages if the proposed mean extension for the level set equation is used. Measurements of the Hausdorff distances exhibit a mean deviation of the surface distances of about 1 to 2 millimeters with only a few outliers. One very important fact is that the manual ground truth segmentation incorporates the isthmus, which is usually not relevant for clinical diagnoses. Therefore, we expect that the results of the semi-automatic algorithms are closer to the actual clinically relevant thyroid volume measurements. We have shown that the previously published demand for a correction of the ellipsoid formula is justified [28]. In the article, the presented algorithms are not fully automatic. However, the approaches do not require more interaction than specifying a few seed structures for the initialization of the level sets and minor adjustments of the edge strength parameters. Although the proposed segmentation is not yet executable in real-time, the computation time of less than 5 minutes still makes it applicable for research and post-processing operations. The statistics and models for noise and speckle reduction and image quality enhancement have to be studied more extensively in order to achieve better pre-processing results. The segmentation may also benefit from the integration of statistical shape information [37], prior knowledge or other kinds of regularization (e.g. 3-D mass-spring model [38]). It has to be investigated if the proposed segmentation approaches are suitable for the analysis of images from patients with different pathologies (e.g. cysts or 
nodules). These pathological structures may lead to additional amplitude variations within the observed thyroid images and pose a challenge to edge-based segmentation algorithms.

\section{CONCLUSION}

Typically, physicians use 2-D US for the examination of thyroid glands. We described how 3-D US together with appropriate image processing techniques may allow for more accurate thyroid measurements. We presented a processing chain for the acquired 3-D US volumes that incorporates intensity correction, filtering and segmentation of thyroid lobes. Besides the time-gain compensation and acquisition parameters of the US machine, posterior structures within the images have been contrast enhanced by an additional ramp filtering. Several other pre-processing algorithms for noise and speckle reduction and edge preservation have been extensively evaluated. Two favorite anisotropic diffusion filters have been combined with level set based segmentation algorithms and applied to ten 3-D thyroid lobe images. The sensitivity of the segmentation was around $75 \%$, the specificity at approximately 97\%. The mean Hausdorff distance of less than $3 \mathrm{~mm}$ is desirable for clinical use.

\section{ACKNOWLEDGMENTS}

The authors would like to thank Prof. Dr. med. R. L. Schild for providing the 3-D US device and for the image acquisition. The authors are also thankful to HipGraphics for providing the volume rendering software InSpace and to G. Wolz and PD Dr. med. M. Grunewald for their time and effort.

\section{REFERENCES}

[1] C. Reiners, K. Wegscheider, H. Schicha, P. Theissen, P. Vaupel, R. Wrbitzky, and P.-M. Schumm-Draeger, "Prevalence of thyroid disorders in the working population of Germany: Ultrasonography screening in 96,278 unselected employees," Thyroid, vol. 14, no. 11, pp. 926-932, Nov. 2004.

[2] S. Ezzat, D. A. Sarti, D. R. Cain, and G. D. Braunstein, "Thyroid incidentalomas: prevalence by palpation and ultrasonography," Archives of internal medicine, vol. 154, no. 16, pp. 1838-1840, Aug. 1994.

[3] L. Hegedüs, S. J. Bonnema, and F. N. Bennedbaek, "Management of simple nodular goiter: Current status and future perspectives," Endocrine Reviews, vol. 24, no. 1, pp. 102-132, Feb. 2003.

[4] H. J. Baskin, "Thyroid ultrasound - just do it," Thyroid, vol. 14, no. 2, pp. 91-92, Feb. 2004.

[5] L. Hegedüs, "Thyroid ultrasound as a screening tool for thyroid disease," Thyroid, vol. 14, no. 11, pp. 879-880, Nov. 2004.

[6] V. F. H. Brauer, P. Eder, K. Miehle, T. D. Wiesner, H. Hasenclever, and R. Paschke, "Interobserver variation for ultrasound determination of thyroid nodule volumes," Thyroid, vol. 15, no. 10, pp. 1169-1175, Oct. 2005.

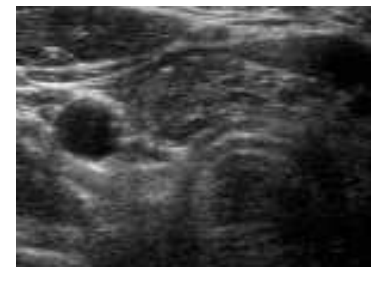

(a)

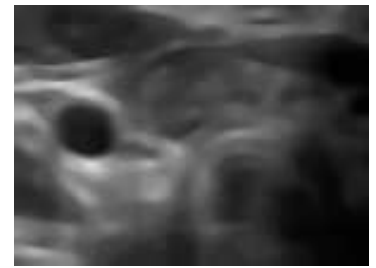

(b)
Fig. 8. 8(a): Original image of thyroid gland lobe number two; 8(b): Preprocessed image with ramp and $\mathrm{CF}$.
TABLE IV

HAUSDORFF DistancES (HD) BETWEEN THE MANUAL (M) AND THE SEMI-AUTOMATIC APPROACH (S), THE LAST TWO COLUMNS SHOW THE MEAN DISTANCE ERROR BETWEEN THE TWO SEGMENTATIONS. THE POSSIBLE COMBINATIONS OF METHODS ARE: \#1: RAMP\&CF, GAC; \#2: RAMP\&GAD, GAC; \#3: RAMP\&CF, GACM; \#4: RAMP\&GAD, GACM.

\begin{tabular}{|c|c|c|c|c|c|}
\hline \multirow{2}{*}{$\begin{array}{l}\text { Data } \\
\text { set no. }\end{array}$} & \multirow[t]{2}{*}{ Comb. } & \multicolumn{2}{|c|}{ Directed HD } & \multicolumn{2}{|c|}{ Mean HD } \\
\hline & & $h(S, M)$ & $h(M, S)$ & $\overline{h(S, M)}$ & $\overline{h(M, S)}$ \\
\hline \multirow{4}{*}{1} & $\# 1$ & 6.14 & 9.49 & 1.82 & 2.07 \\
\hline & $\# 2$ & 5.84 & 9.38 & 1.65 & 2.24 \\
\hline & $\# 3$ & 6.36 & 9.35 & 1.83 & 2.08 \\
\hline & $\# 4$ & 5.60 & 9.63 & 1.53 & 2.30 \\
\hline \multirow{4}{*}{2} & $\# 1$ & 12.83 & 14.84 & 3.21 & 3.46 \\
\hline & $\# 2$ & 12.66 & 15.09 & 3.19 & 3.59 \\
\hline & $\# 3$ & 12.77 & 14.84 & 3.22 & 3.47 \\
\hline & $\# 4$ & 12.66 & 15.09 & 3.17 & 3.60 \\
\hline \multirow{4}{*}{3} & $\# 1$ & 3.61 & 8.00 & 0.87 & 1.61 \\
\hline & $\# 2$ & 3.54 & 7.92 & 0.91 & 1.61 \\
\hline & $\# 3$ & 3.63 & 7.98 & 0.89 & 1.60 \\
\hline & $\# 4$ & 3.54 & 8.03 & 0.88 & 1.65 \\
\hline \multirow{4}{*}{4} & $\# 1$ & 4.36 & 7.14 & 0.98 & 1.11 \\
\hline & $\# 2$ & 4.28 & 7.30 & 0.94 & 1.11 \\
\hline & $\# 3$ & 4.39 & 7.18 & 0.97 & 1.12 \\
\hline & $\# 4$ & 4.28 & 7.35 & 0.92 & 1.11 \\
\hline \multirow{4}{*}{5} & $\# 1$ & 4.97 & 5.91 & 1.19 & 1.25 \\
\hline & $\# 2$ & 4.86 & 5.88 & 1.20 & 1.26 \\
\hline & $\# 3$ & 4.82 & 6.09 & 1.12 & 1.29 \\
\hline & $\# 4$ & 4.76 & 6.06 & 1.15 & 1.28 \\
\hline \multirow{4}{*}{6} & $\# 1$ & 4.13 & 8.25 & 1.19 & 1.59 \\
\hline & $\# 2$ & 4.10 & 8.22 & 1.18 & 1.57 \\
\hline & \#3 & 4.13 & 8.26 & 1.18 & 1.60 \\
\hline & $\# 4$ & 4.10 & 8.22 & 1.18 & 1.57 \\
\hline \multirow{4}{*}{7} & $\# 1$ & 3.51 & 7.86 & 1.11 & 1.78 \\
\hline & $\# 2$ & 3.46 & 8.06 & 1.05 & 1.82 \\
\hline & \#3 & 3.74 & 7.79 & 1.08 & 1.72 \\
\hline & $\# 4$ & 3.46 & 7.98 & 1.04 & 1.77 \\
\hline \multirow{4}{*}{8} & $\# 1$ & 3.73 & 4.75 & 0.92 & 1.06 \\
\hline & $\# 2$ & 3.87 & 5.01 & 0.96 & 1.13 \\
\hline & \#3 & 3.65 & 4.86 & 0.90 & 1.07 \\
\hline & \#4 & 3.41 & 5.55 & 0.90 & 1.24 \\
\hline \multirow{4}{*}{9} & $\# 1$ & 4.15 & 3.51 & 0.96 & 0.83 \\
\hline & $\# 2$ & 3.61 & 3.61 & 0.96 & 0.81 \\
\hline & \#3 & 4.13 & 3.50 & 0.95 & 0.82 \\
\hline & \#4 & 3.60 & 3.59 & 0.96 & 0.80 \\
\hline \multirow{4}{*}{10} & $\# 1$ & 3.93 & 7.06 & 1.06 & 1.35 \\
\hline & $\# 2$ & 3.87 & 6.44 & 1.02 & 1.23 \\
\hline & \#3 & 4.07 & 7.77 & 1.00 & 1.48 \\
\hline & $\# 4$ & 3.87 & 6.46 & 1.01 & 1.22 \\
\hline
\end{tabular}

[7] S. Schlögl, E. Werner, M. Lassmann, J. Terekhova, S. Muffert, S. Seybold, and C. Reiners, "Techniques in thyroidology: The use of threedimensional ultrasound for thyroid volumetry," Thyroid, vol. 11, no. 6, pp. 569-574, June 2001.

[8] G. Xiao, M. Brady, J. A. Noble, and Y. Zhang, "Segmentation of ultrasound B-mode images with intensity inhomogeneity correction," IEEE Transactions on Medical Imaging, vol. 21, no. 1, pp. 48-57, Jan. 2002.

[9] R. N. Czerwinski, D. L. Jones, and W. D. O’Brien Jr., "Line and boundary detection in speckle images," IEEE Transactions on Image Processing, vol. 7, no. 12, pp. 1700-1714, Dec. 1998.

[10] — "Detection of lines and boundaries in speckle images - application to medical ultrasound," IEEE Transactions on Medical Imaging, vol. 18, no. 2, pp. 126-136, Feb. 1999.

[11] H. Shi and X. Wu, "Despeckle filtering in medical ultrasound imaging," http://homepages.cae.wisc.edu//ece533/project/f03/shiwu.pdf, Dec. 2003.

[12] S. Fu, Q. Ruan, W. Wang, and Y. Li, "Adaptive anisotropic diffusion for ultrasonic image denoising and edge enhancement," International Journal of Signal Processing, vol. 1, no. 4, pp. 282-290, 2004.

[13] K. Krissian, K. Vosburgh, R. Kikinis, and C.-F. Westin, "Anisotropic diffusion of ultrasound constrained by speckle noise model," Department of Radiology, Brigham and Women's Hospital, Harvard Medical School, 
Laboratory of Mathematics in Imaging, Tech. Rep. 4, Oct. 2004.

[14] Y. Yu and S. T. Acton, "Speckle reducing anisotropic diffusion," IEEE Transactions on Image Processing, vol. 11, no. 11, pp. 1260-1270, Nov. 2002.

[15] P. B. Calóope, F. N. S. de Medeiros, R. C. P. Marques, and R. C. S. Costa, "A comparison of filters for ultrasound images," in Telecommunications and Networking - ICT 2004, de Souza, Ed., 2004.

[16] C. Tauber, H. Batatia, and A. Ayache, "A robust speckle reducing anisotropic diffusion," in 11th IEEE International Conference on Image Processing (ICIP 2004), IEEE, Ed., vol. 1. Singapore, Republic of Singapore: IEEE Signal Processing Society, Oct. 2004, pp. 247-250.

[17] N. Betrouni, J. Rousseau, M. Vermandel, D. Pasquier, and S. Maouche, "Automatic segmentation of prostate boundaries from abdominal ultrasound images using priori knowledge," in IEEE International Symposium on Biomedical Imaging: Nano to Macro, IEEE, Ed. IEEE Computer Society Press, Apr. 2004, pp. 496-499.

[18] M. Fieberg, "Registrierung zwei- und dreidimensionaler multimodaler medizinischer Bilder - angewendet für Ultraschall- und Magnetresonanzbilder," Master's thesis, Institut für Informatik III, Rheinische FriedrichWilhelms-Universität, Bonn, Germany, Jan. 1999.

[19] N. Hu, D. B. Downey, A. Fenster, and H. M. Ladak, "Prostate boundary segmentation from 3d ultrasound images," Medical Physics, vol. 30, no. 7 , pp. $1648-1659$, July 2003 .

[20] B. Chiu, G. H. Freeman, M. M. A. Salama, and A. Fenster, "Prostate segmentation algorithm using dyadic wavelet transform and discrete dynamic contour," Physics in Medicine and Biology, vol. 49, no. 21, pp. 4943-4960, Nov. 2004.

[21] D.-R. Chen, R.-F. Chang, W.-J. Wu, W. K. Moon, and W.-L. Wu, "3-d breast ultrasound segmentation using active contour model," Ultrasound in Medicine and Biology, vol. 29, no. 7, pp. 1017-1026, July 2003.

[22] C. Baillard, C. Barillot, and P. Bouthemy, "Robust adaptive segmentation of $3 \mathrm{~d}$ medical images with level sets," Institut national de recherche en informatique et en automatique (INRIA), Le Chesnay Cedex, France, Tech. Rep. 4071, Nov. 2000.

[23] P. Abolmaesumi and M. R. Sirouspour, "An interacting multiple model probabilistic data association filter for cavity boundary extraction from ultrasound images," IEEE Transactions on Medical Imaging, vol. 23, no. 6, pp. 772-784, June 2004.

[24] A. Sarti, C. Corsi, E. Mazzini, and C. Lamberti, "Maximum likelihood segmentation of ultrasound images with rayleigh distribution," IEEE Transactions on Ultrasonics, Ferroelectrics and Frequency Control, vol. 52, no. 6, pp. 947-960, June 2005.

[25] H. M. Overhoff, S. Maas, T. Cornelius, and S. Hollerbach, "Visualisierung anatomischer Strukturen von Oberbauchorganen mittels automatisch segmentierter 3D-Ultraschallbildvolumina. Ergebnisse einer Pilotstudie," in Bildverarbeitung für die Medizin (BVM), ser. CEUR Workshop Proceedings, T. Wittenberg, P. Hastreiter, U. Hoppe, H. Handels, A. Horsch, and H.-P. Meinzer, Eds., vol. 80. Erlangen, Germany: CEUR-WS.org, Mar. 2003, pp. 358-362.

[26] A. Lyshchik, V. Drozd, and C. Reiners, "Techniques in thyroidology accuracy of three-dimensional ultrasound for thyroid volume measurement in children and adolescents," Thyroid, vol. 14, no. 2, pp. 113-120, Feb. 2004.

[27] P. Andermann, S. Schlögl, U. Mäder, M. Luster, M. Lassmann, and C. Reiners, "Intra- and interobserver variability of thyroid volume measurements in healthy adults by $2 \mathrm{~d}$ versus $3 \mathrm{~d}$ ultrasound," Nuklearmedizin, vol. 46, no. 1, pp. 1-7, 2007.

[28] P. Reinartz, O. Sabri, M. Zimny, B. Nowak, U. Cremerius, K. Setani, and U. Büll, "Thyroid volume measurement in patients prior to radioiodine theraphy: Comparison between three-dimensional magnetic resonance imaging and ultrasonography," Thyroid, vol. 12, no. 8, pp. 713-717, Aug. 2002.

[29] B. Nygaard, T. Nygaard, M. Court-Payen, L. Ingeman Jensen, P. SœJensen, K. G. Nielsen, M. Fugl, and L. Hegedüs, "Thyroid volume measured by ultrasonongraphy and CT," Acta Radiologica, vol. 43, no. 3 , pp. 269-274, May 2002.

[30] C. Kotropoulos, X. Magnisalis, I. Patis, and M. G. Strintzis, "Nonlinear ultrasonic image processing based on signal-adaptive filters and selforganizing neural networks," IEEE Transactions on Medical Imaging, vol. 3, no. 1, pp. 65-77, Jan. 1994.

[31] J. A. Sethian, Level Set Methods and Fast Marching Methods - Evolving Interfaces in Computational Geometry, Fluid Mechanics, Computer Vision and Materials Science, 2nd ed. Cambridge, United Kingdom: Cambridge University Press, June 1999.

[32] J. Weickert, "Applications of nonlinear diffusion in image processing and computer vision," in Acta Mathematica Universitatis Comenianae,
J. Kacur, K. Mikula, and D. Sevcovic, Eds., vol. 70, no. 1, Sept. 2001, pp. 33-50.

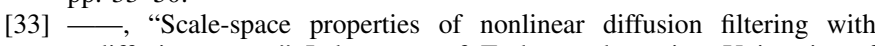
a diffusion tensor," Laboratory of Technomathematics, University of Kaiserslautern, Tech. Rep. 110, Oct. 1994.

[34] V. Caselles, R. Kimmel, and G. Sapiro, "Geodesic active contours," in Fifth International Conference on Computer Vision (ICCV), IEEE, Ed. IEEE Computer Society Press, June 1995, pp. 694-699.

[35] R. Malladi, J. A. Sethian, and B. C. Vemuri, "Shape modeling with front propagation: A level set approach," IEEE Transactions on Pattern Analysis and Machine Intelligence, vol. 17, no. 2, pp. 158-175, Feb. 1995.

[36] L. Grady, "Random walks for image segmentation," IEEE Transactions on Pattern Analysis and Machine Intelligence, vol. 28, no. 11, pp. 17681783, Nov. 2006.

[37] M. Leventon, W. Grimson, and O. Faugeras, "Statistical shape influence in geodesic active contours," in IEEE Conference on Computer Vision and Pattern Recognition (CVPR), IEEE, Ed., vol. 1. IEEE Computer Society Press, June 2000, pp. 1316-1323.

[38] J. Dornheim, L. Dornheim, B. Preim, K. D. Tönnies, I. Hertel, and G. Strauss, "Stable 3d mass-spring models for the segmentation of the thyroid cartilage," Institut für Simulation und Graphik, Otto-vonGuericke-Universität Magdeburg, Tech. Rep., 2005. 